Pacific Journal of Mathematics

INTEGRAL REPRESENTATIONS OF ALGEBRAIC
COHOMOLOGY CLASSES ON HYPERSURFACES 


\section{INTEGRAL REPRESENTATIONS OF ALGEBRAIC COHOMOLOGY CLASSES ON HYPERSURFACES}

\section{Edith Stevenson}

Let $f_{\lambda}(X)=\sum_{i=1}^{n+1} X_{i}^{d}+h_{\lambda}(X)$ where $h_{\lambda}(X)$ is the generic form of degree $d$ in $n+1$ variables over $C$. The main theorem of this paper is that certain exponential integrals associated to the projective hypersurface $X_{\lambda}$ defined by the vanishing of $f_{\lambda}(X)$ have regular singularities. The main ingredients in the proof are:

1. Katz's identification of certain monomial spaces (first studied by Dwork) in terms of the middle-dimensional cohomology of a variety related to $X_{\lambda}$ and

2. Griffiths' theorem, which states that periods on an algebraic variety have regular singularities.

By essentially the same methods, an upper bound on the order of logarithmic growth of the integrals is determined.

Also, an example is given to show the relation of periods on a family of cubic curves to hypergeometric functions.

Introduction. The main theorem of this paper is that certain exponential integrals associated to a family of hypersurfaces have regular singularities. The major ingredients in the proof are:

(1) Katz's thesis [4], which provides cohomological meaning to certain polynomial spaces first studied by Dwork and, as a consequence, provides cohomological meaning to the integrals in question, and

(2) Griffiths' theorem [6], which says that the Picard-Fuchs equations satisfied by periods on an algebraic variety have regular singularities.

Let $f_{\lambda}(X)=X_{1}^{d}+\cdots+X_{n+1}^{d}+h_{\lambda}(X)$, (a diagonal form plus a perturbation term $\left.h_{\lambda}(X)\right)$ where $h_{\lambda}(X)=\sum_{i=1}^{\nu} \lambda_{i} X^{w^{(i)}}$, the generic form of degree $d$ in $n+1$ variables over $C$. We are interested in the family of complex projective hypersurfaces $X_{\lambda}$ defined by the vanishing of $f_{\lambda}(X)$. The integrals associated to the family $X_{\lambda}, \lambda \in C^{\nu}$, come about as follows:

Let $K=\boldsymbol{Q}(\lambda)$. Let $\mathscr{L}$ be the $K$-span of all monomials $t^{v}=$ $t_{1}^{v_{1}} \cdots t_{n+1}^{v_{n+1}}$ where $\sum_{i=1}^{n+1} v_{i} \equiv 0(\bmod d)$ and $v_{i} \geqq 0, i=1, \cdots, n+1$. Let $M_{d}^{n+1}$ denote $n+1$ copies of the group $M_{d}$ of $d$ th roots of unity and let $\omega=e^{2 \pi i / d}$. Then for $\zeta \in M_{d}^{n+1}$, we assign to each monomial $t^{v} \in \mathscr{L}$ the multiple integral

$$
\varphi_{\zeta}\left(t^{v}\right)=\int_{\zeta \infty}^{\zeta \omega \infty} t^{v} \exp \left(-f_{\lambda}(t)\right) \frac{d t}{t}
$$

where $d t / t=d t_{1} / t_{1} \cdots d t_{n+1} / t_{n+1}$ and the region of integration is the 
$(n+1)$-fold product of limits as $\rho \rightarrow 0$ along paths of the type:

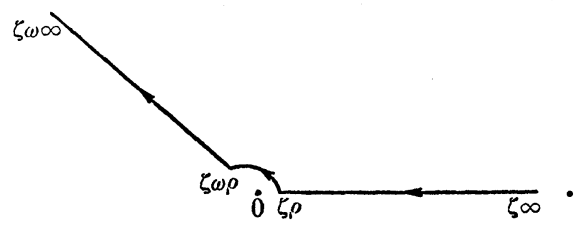

To the subspace $\mathscr{L}^{s}$ of $\mathscr{L}$ consisting of monomials $t^{v}$ which are divisible by all variables (i.e., $v_{i}>0, i=1, \cdots, n+1$ ), we associate the integrals

$$
\hat{\varphi}_{\zeta}\left(t^{v}\right)=\int_{0}^{\zeta \infty} t^{v} \exp \left(-f_{\lambda}(t)\right) \frac{d t}{t}
$$

where, as before, $\zeta \in M_{d}^{n+1}$.

It is easily shown that both of these classes of integrals have nonzero radii of convergence. (See the note after Theorem 2.) The main result of this paper is that the integrals $\left({ }^{*}\right)$ and $\left({ }^{* *}\right)$ have regular singularities as functions of $\lambda$, which is perhaps surprising since the integrands involve exponentials and one might reasonably expect the integrals to have exponential growth. The fact that $f_{\lambda}(t)$ is taken to be homogeneous is crucial as is shown in $\$ 6$.

Since periods are integrals of differential forms over cycles on a variety, a direct proof of the regularity of the integrals (*) and $\left({ }^{* *}\right)$ would involve showing that the integrands are algebraic cohomology classes on a suitable variety and therefore (after explaining the regions of integration) the integrals are periods. Regularity would follow from Griffiths' result. We do not use this method of proof but Chapter 4 of [7] provides a heuristic explanation of why the integrands should correspond to cohomology classes on a variety related to $X_{2}$ (following the theory of Monsky as explained in [3]).

Instead, we proceed by identifying the space of integrals $\left(^{*}\right)$, $\mathscr{J}_{5} \cong \mathscr{L} / \operatorname{ker} \varphi$, in terms of the middle dimensional cohomology of the variety $X_{\lambda}^{\phi}$, the subvariety of $X_{\lambda}^{\phi}$ where no coordinate vanishes. (The fact that this is the variety of interest is suggested by the $d t / t$ in the integrands of $\left(^{*}\right)$.) The key is to interpret $\operatorname{ker} \varphi$ in terms of the differential operators

$$
D_{i}=\exp \left(f_{\lambda}(X)\right) \circ X_{i} \frac{\partial}{\partial X_{i}} \circ \exp \left(-f_{\lambda}(X)\right), \quad i=1, \cdots, n+1,
$$

which act on $\mathscr{L}$. The motivation for this is that spaces ressembling the quotient space $\mathscr{C}_{\lambda}=\mathscr{L} / \Sigma D_{i, 2} \mathscr{L}$ have cohomological meaning, as 
is shown by Katz in his thesis [4]. Katz's results enable us to interpret the space $\mathscr{V}_{\lambda}$ in terms of the middle dimensional cohomology of the variety $X_{i}^{\phi}$.

Since $\varphi$ is surjective by definition and since $\Sigma D_{i, \lambda} \mathscr{L} \subset \operatorname{ker} \varphi$, it follows that $\varphi: \mathscr{\mathscr { V }}_{\lambda} \rightarrow \mathscr{F}_{\zeta}$ is surjective, and hence that each integral $I(\lambda) \in \mathscr{F}_{\zeta}$ may be regarded as the image under $\phi$ of a cohomology class on $X_{\lambda}^{\phi}$.

The next step is to prove that the following diagram commutes:

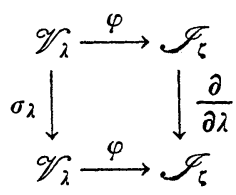

where $\sigma_{\lambda}$ is a connection on $\mathscr{V}_{\lambda}$ which corresponds to $\partial / \partial \lambda$ acting on $H^{n-1}\left(X_{\lambda}^{\phi}, K\right)$, the middle dimensional cohomology of $X_{\lambda}^{\phi}$. Hence, the differential equations satisfied by the integrals in $\mathscr{J}$ are given by $\sigma_{\lambda}$ acting on $\mathscr{\mathscr { T }}$, which in turn are given by $\partial / \partial \lambda$ acting on $H^{n-1}\left(X^{\phi}, K\right)$. By Griffith's theorem, these differential equations have regular singularities so the regularity theorem follows for the integrals $\left(^{*}\right)$.

To draw similar conclusions about the integrals $\left({ }^{* *}\right)$, it would suffice to give cohomological meaning to the space $\mathscr{V}_{\lambda}^{s}=\mathscr{L}^{s} / \Sigma D_{i, \lambda} \mathscr{L}^{s}$. Rather than do this directly, we use the isomorphism $\Theta_{\lambda}$ constructed by Dwork between $\left(\mathscr{V}_{\lambda}\right)^{*}$, the dual space of $\mathscr{V}_{\lambda}$, and $\mathscr{\mathscr { C }}_{\lambda}^{s}$. The basic idea is as follows: we know that the integrals $\left({ }^{*}\right)$, viewed on the space $\mathscr{V}_{\lambda}$, satisfy a system of differential equations with regular singularities and we want to see how the system is changed under $\Theta_{\lambda}$. This is equivalent to studying the behavior of $\sigma_{\lambda}^{*}$, the connection on $\mathscr{V}_{\lambda}^{*}$ dual to $\sigma_{\lambda}$, under $\Theta_{\lambda}$. We show that under $\Theta_{\lambda}$ the matrix of the system of differential equations is transposed. The conclusion is that the integrals $\left({ }^{* *}\right)$ satisfy a system of differential equations with regular singularities.

After proving Theorem 2, we use essentially the same techniques to prove a theorem (Theorem 3) on the order of logarithmic singularity occurring in the integrals $\left({ }^{*}\right)$ and $\left({ }^{* *}\right)$.

The original proof of the regularity and logarithmic growth theorems [7] used Dwork's deformation theory, a $p$-adic theory, to derive an expression for the integrals $\left(^{*}\right)$ as sums of periods on $X_{\lambda}^{\phi}$. Although the proof given here has the advantage of being much shorter and of using less machinery, the original proof has the advantage of being more explicit. For example, in the special case where the defining form for the family of hypersurfaces has only one perturbing term (and hence is of the form $f_{\lambda}(X)=\sum_{i=1}^{n+1} X_{i}^{d}+\lambda X^{w}$ ), the formula relating periods and integrals may be applied to express 
the periods on $X_{\lambda}^{\phi}$ in terms of hypergeometric functions. In this case, the results about the growth of the integrals follow from classical hypergeometric theory. (Griffiths' theorem needn't be used.)

This paper is organized as follows:

$\S 1$ contains preliminaries on Dwork's spaces and a cohomological interpretation of $\mathscr{\mathscr { \lambda }}$.

$\S 2$ contains the statement and proof of the main theorem (as outlined above).

$\S 3$ contains results on the order of logarithmic singularity of the integrals.

$\S 4$ outlines briefly the original method of proof of the regularity theorem and gives the formula for the integrals $\left(^{*}\right)$ in terms of periods on $X_{\lambda}^{\phi}$.

$\S 5$ contains an example showing the connection with hypergeometric theory, and

$\S 6$ concludes with an example showing the necessity of the hypothesis that the defining form $f_{\lambda}(X)$ be homogeneous.

This paper is abstracted from my $\mathrm{Ph}$. D. thesis written under the direction of Bernard Dwork. At this time, I would like to express my gratitude to him for his generosity with both his time and his ideas.

1. In this section we are interested in the cohomological interpretation of a certain polynomial space which Dwork constructed for a family of complex projective hypersurfaces in connection with his work on zeta functions.

Let $f_{\lambda}(X)=f(X)=X_{1}^{d}+\cdots+X_{n+1}^{d}+h_{\lambda}(X)$ where $h_{\lambda}(X)=$ $\sum_{i=1}^{\nu} \lambda_{i} X^{w(i)}$ is the generic form of degree $d$ in $n+1$ variables over C. Let $K=Q\left(\lambda_{1}, \cdots, \lambda_{\nu}\right)$. Let $\mathscr{L}$ be the subspace of $K[X]=$ $K\left[X_{1}, \cdots, X_{n+1}\right]$ spanned by $\left\{X^{w}=X_{1}^{w_{1}} \cdots X_{n+1}^{w_{n+1}} \mid \sum_{i=1}^{n+1} w_{i} \equiv 0(\bmod d)\right.$ and $\left.w_{i} \geqq 0, i=1, \cdots, n+1\right\}$, and let $\mathscr{L}^{s}$ be the subspace of $\mathscr{L}$ spanned by all $X^{w}$ satisfying the further condition: $w_{i}>0, i=1$, $\cdots, n+1$. Thus $\mathscr{L}^{s}$ is the $K$-span of the monomials divisible by all the variables.

Given $f(X) \in K[X]$, let $D_{i}$ be the twisted differential operator on $\mathscr{L}$ defined by

$$
\begin{aligned}
D_{i}=\exp (-\pi f(X)) \circ X_{i} \frac{\partial}{\partial X_{i}} \circ \exp (\pi f(X))=X_{i} \frac{\partial}{\partial X_{i}}+\pi X_{i} \frac{\partial f}{\partial X_{i}}, \\
i=1, \cdots, n+1,
\end{aligned}
$$

where $\pi$ is some nonzero constant. Without loss of generality we may take $\pi$ to be -1 . (References to papers of Dwork or Katz should be modified accordingly.) 
Both $\mathscr{L}$ and $\mathscr{L}^{8}$ are stable under $D_{i}$ so we can form the quotient spaces $\mathscr{V}_{i}=\mathscr{L} / \sum_{i=1}^{n+1} D_{i, \lambda} \mathscr{L}$ and $\mathscr{V}_{i}^{s}=\mathscr{L}^{s} / \sum_{i=1}^{n+1} D_{i, \lambda} \mathscr{L}^{s}$. These are finite dimensional $K$-vector spaces and in the case where the hypersurface $X_{\lambda}$ defined by the vanishing of $f_{\lambda}(X), \lambda \in C^{\nu}$, is nonsingular and in general position, they both have dimension $d^{n}[1]$. The condition that $X_{\lambda}$ be nonsingular and in general position means that the forms $X_{i}\left(\partial f / \partial X_{i}\right), i=1, \cdots, n+1$, have no common zero. By elimination theory, there is a polynomial $R(\lambda)$ with integral coefficients such that $X_{\lambda}$ is nonsingular and in general position precisely when $\lambda$ is not a zero of $R(\lambda)$. Thus, outside the subvariety $U$ defined by the vanishing of $R(\lambda), \mathscr{T}_{\lambda}$ and $\mathscr{\mathscr { T }}_{\lambda}^{s}$ have dimension $d^{n}$.

Let $\mathscr{A}=\left\{w=\left(w_{1}, \cdots, w_{n+1}\right) \in Z^{n+1} \mid \sum_{i=1}^{n+1} w_{i} \equiv 0(d)\right.$ and $0 \leqq w_{i}<d$, $i=1, \cdots, n+1\}$ and let $\mathscr{C}^{\prime}=\left\{w=\left(w_{1}, \cdots, w_{n+1}\right) \in \mathbb{Z}^{n+1} \mid \sum_{i=1}^{n+1} w_{i} \equiv 0(d)\right.$ and $\left.0<w_{i} \leqq d, i=1, \cdots, n+1\right\}$. Then for $\left(\lambda_{1}, \cdots, \lambda_{\nu}\right) \in C^{\nu}-U$, $\left\{X^{w}\right\}_{w \in \mathscr{M}}$ is a basis for $\mathscr{C}_{\lambda}$ and $\left\{X^{w}\right\}_{w \in \mathscr{\varkappa}}$, is a basis of $\mathscr{V}_{\lambda}^{:}[1]$.

With these preliminaries, we turn to the cohomological interpretation of $\mathscr{T}_{\lambda}=\mathscr{L} / \Sigma D_{i, \lambda} \mathscr{L}$. Let $X_{\lambda}^{\phi}$ denote the open subset of $X_{\lambda}$ where no coordinate vanishes and let $\boldsymbol{P}_{n+1}^{n}$ denote the open subset of $\boldsymbol{P}^{n}$ where no coordinate vanishes. Let $\mathscr{L}^{1}=\mathscr{L}-\{1\}$. In his thesis [4], Katz constructs an isomorphism

$$
\mathscr{R}: \mathscr{L}^{1} / \sum_{i=1}^{n+1} D_{i} \mathscr{L}^{1} \longrightarrow H^{n}\left(\boldsymbol{P}_{n+1}^{n}-X^{\phi}\right)
$$

by defining $\mathscr{R}\left(X^{w}\right)=\left(w_{0}-1\right) !\left(X^{w} / f^{w_{0}}\right)\left(d\left(X_{1} / X_{n+1}\right) / X_{1} / X_{n+1}\right) \wedge \cdots \wedge$ $\left(d\left(X_{n} / X_{n+1}\right) / X_{n} / X_{n+1}\right)$ where $H^{n}\left(\boldsymbol{P}_{n+1}^{n}-X^{\phi}\right)$ denotes the $n$ dimensional cohomology group $H^{n}\left(\boldsymbol{P}_{n+1}^{n}-X^{\phi}, K\right)$ and $w_{0}=(1 / d) \sum_{i=1}^{n+1} w_{i}$. He computes the dimension of $H^{n}\left(\boldsymbol{P}_{n+1}^{n}-X^{\phi}\right)$ to be $d^{n}+n$.

Let $\mathscr{W}_{\lambda}=\mathscr{V}_{\lambda}-\{1\}=\mathscr{L}^{1} / \Sigma D_{i, \lambda} \mathscr{L}$. Then the following sequence is exact:

$$
0 \longrightarrow \Sigma D_{i} \mathscr{L} \mid \Sigma D_{i} \mathscr{L}^{1} \longrightarrow \mathscr{L}^{1} / \Sigma D_{i} \mathscr{L}^{1} \longrightarrow \mathscr{W}_{\lambda} \longrightarrow 0,
$$

so $\mathscr{W}_{\lambda}$ is a factor space of Katz's space $\mathscr{L}^{1} / \Sigma D_{i} \mathscr{L}^{1}$.

Lemma 1. $\left\{X^{w}\right\}_{\substack{\omega \in \neq(1, \cdots, \cdots, 0\} \\ w \neq 1}} \cup\left\{X_{i}\left(\partial f / \partial X_{i}\right)\right\}_{i=1}^{n+1}$ is a basis for $\mathscr{L}^{1} / \Sigma D_{i} \mathscr{L}^{1}$.

Proof. Since $\mathscr{L}$ differs from $\mathscr{L}^{1}$ by $\{1\}$, a basis for $\Sigma D_{i} \mathscr{L} / \Sigma D_{i} \mathscr{L}^{1}$, the kernel of the projection mapping, is $\left\{D_{i}(1)\right\}_{i=1}^{n+1}=\left\{X_{i}\left(\partial f / \partial X_{i}\right)\right\}_{i=1}^{n+1}$.

We now describe the additional cohomology classes in $\mathscr{L}^{1} / \Sigma D_{i} \mathscr{L}^{1}$, i.e., those corresponding to the forms $X_{i}\left(\partial f / \partial X_{i}\right)$ under the Katz $\mathscr{R}$ map. To do this we define a connection on $\mathscr{L}^{1} / \Sigma D_{i} \mathscr{L}^{1}$ which corresponds to the derivation $\partial / \partial \lambda$ on $H^{n}\left(\boldsymbol{P}_{n+1}^{n}-X^{\phi}\right)$ under the map $\mathscr{R}$ (Lemma 2), and then show that under the connection the $X_{i}\left(\partial f / \partial X_{i}\right)$ 's 
are taken to $\Sigma D_{i} \mathscr{L}^{1}$ and thus may be thought of as constant cohomology classes (since taking their $\lambda$ derivatives makes them exact).

Let $\sigma_{\lambda_{i}}=\exp (-\pi f(X)) \circ \partial / \partial \lambda_{i} \circ \exp (\pi f(X))=\partial / \partial \lambda_{i}+\pi\left(\partial f / \partial \lambda_{i}\right), i=$ $1, \cdots, \nu$, where $\pi$ is a constant which we shall take to be -1 and where $\partial \lambda_{j} / \partial \lambda_{i}=\delta_{i j}$. Then $\sigma_{\lambda_{i}}, i=1, \cdots, \nu$, is a connection on $\mathscr{L}$ [5] and since it commutes with the $D_{i}$ 's, $i=1, \cdots, n+1$, it is also a connection on $\mathscr{L}^{1} / \Sigma D_{i} \mathscr{L}^{1}$.

LEMma 2. The following diagram commutes:

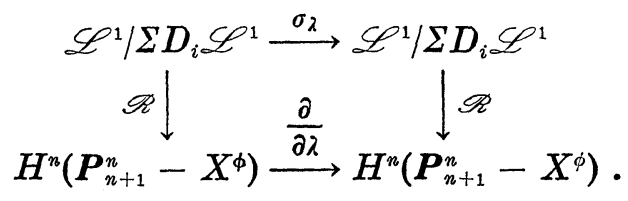

Proof. The proof is a standard computation and will be omitted.

With the understanding that a constant cohomology class is one whose $\lambda$ derivative is exact, we have the following result:

THEOREM 1. The nonconstant cohomology classes of $H^{n}\left(\boldsymbol{P}_{n+1}^{n}-X^{\phi}\right)$ are contained in $\mathscr{W}_{\lambda}$.

Proof. For $i=1, \cdots, n+1$,

$$
\frac{\partial}{\partial \lambda}\left(\mathscr{R}\left(X_{i} \frac{\partial f}{\partial X_{i}}\right)\right)=\frac{\partial}{\partial \lambda}\left(\mathscr{R} \circ D_{i}(1)\right)=\mathscr{R} \circ \sigma_{\lambda}\left(D_{i}(1)\right) \subset \mathscr{R}\left(D_{i} \mathscr{L}^{1}\right)
$$

so $\partial / \partial \lambda$ applied to the forms defined by $X_{i}\left(\partial f / \partial X_{i}\right), i=1, \cdots, n+1$, gives an exact form, which is the statement of the theorem.

Note. It is not true that all the forms in $\mathscr{W}_{\lambda}$ correspond via $\mathscr{R}$ to nonconstant cohomology classes. For example, let $f(X)=$ $X_{1}^{3}+X_{2}^{3}+X_{3}^{2}-3 \lambda X_{1} X_{2} X_{3}$. Then

$$
\sigma_{\lambda}\left(X_{1} X_{2}^{2}\right)=-D_{1}\left(\frac{\lambda}{1-\lambda^{3}} X_{2} X_{3}^{2}\right)-D_{2}\left(\frac{1}{1-\lambda^{3}} X_{1}^{2} X_{3}\right)-D_{3}\left(\frac{\lambda^{2}}{1-\lambda^{3}} X_{1} X_{2}^{2}\right),
$$

so the cohomology class corresponding to $X_{1} X_{2}^{2}$ is constant.

Theorem 1 provides the necessary cohomological interpretation of $\mathscr{V}_{\lambda}=\mathscr{L} \mid \Sigma D_{i, \lambda} \mathscr{L}: \mathscr{V}_{\lambda}$ may be viewed as containing the nonconstant cohomology classes of $H^{n}\left(\boldsymbol{P}_{n+1}^{n}-X^{\phi}\right)$ together with a class singled out by $\sigma_{\lambda}(1)$.

Note. In his thesis, Katz also shows 


$$
\mathscr{L}^{1} /(f)+\sum_{i=1}^{n+1} D_{i} \mathscr{L}^{1} \cong H^{n-1}\left(X_{i}^{\phi}\right) .
$$

$\mathscr{W}_{\lambda}$ is a factor space of $\mathscr{L}^{1} /(f)+\sum_{i=1}^{n+1} D_{i} \mathscr{L}^{1}$ and an argument similar to the one above shows that $\mathscr{V}_{2}$ may also be viewed as containing the nonconstant cohomology classes of $H^{n-1}\left(X_{\lambda}^{\phi}\right)$ together with a class singled out by $\sigma_{\lambda}(1)$.

2. This section is devoted to the proof of the main theorem:

THEOREM 2. The multiple integrals

$$
I(\lambda)=\int_{\infty}^{\omega \infty}(\zeta t)^{v} \exp \left(-f_{\lambda}(\zeta t)\right) \frac{d t}{t}, \quad v \in \mathscr{A}, \zeta \in M_{d}^{n+1}, \omega=e^{2 \pi \imath / d}
$$

and

$$
\hat{I}(\lambda)=\int_{0}^{\infty}(\zeta t)^{v} \exp \left(-f_{\lambda}(\zeta t)\right) \frac{d t}{t}, \quad v \in \mathscr{L}^{\prime}, \zeta \in M_{d}^{n+1}
$$

have regular singularities as functions of $\lambda$, where the region of integration for $I(\lambda)$ is the $(n+1)$-fold limit as $\rho \rightarrow 0$ along paths of the type:

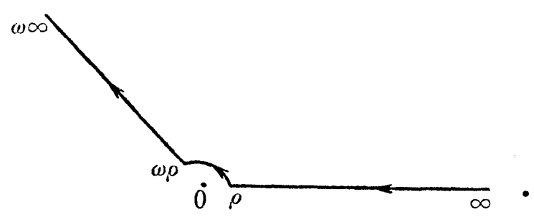

Note. For $\lambda=0$,

and

$$
I(0)=\frac{1}{d^{n+1}} \prod_{i=1}^{n+1} \zeta_{i}^{v_{i}}\left(\omega^{v_{i}}-1\right) \Gamma\left(\frac{v_{i}}{d}\right)
$$$$
\hat{I}(0)=\frac{1}{d^{n+1}} \prod_{i=1}^{n+1} \zeta_{i}^{v_{i}}\left(\frac{v_{i}}{d}\right) .
$$

( $\Gamma$ denotes the gamma function.)

Hence, by the Lebesgue dominated convergence theorem, it follows that both integrals converge in the $\nu$-fold product of complex half planes: $\operatorname{Re}\left(\lambda_{i}\right) \geqq 0, i=1, \cdots, \nu$. The domain of convergence is actually larger. In [7] it is shown that the product of half planes in which the integrals converge is given explicitly by the condition:

$\operatorname{Re}\left(\zeta^{w^{(i)}} \lambda_{i}\right)>-\alpha_{i} / \gamma_{i}, i=1, \cdots, \nu$ where $\gamma_{i}=d /\left(w^{(i)}\right)^{w^{(i)} / d}$ and where the $\alpha_{i}$ 's are positive real numbers satisfying the condition $\sum_{i=1}^{\nu} \alpha_{i}=1$. (Recall that $f_{\lambda}(X)=X_{1}^{d}+\cdots+X_{n+1}^{d}+\sum_{i=1}^{\nu} \lambda_{i} X^{w^{(i)}}$.) 
Proof of Theorem 2. We begin with the collection $\mathscr{F}_{\zeta}\left(\zeta \in M_{d}^{n+1}\right)$ of integrals of the type $\left({ }^{*}\right)$ (regarded as functions of $\lambda$ ): for each $\zeta \in M_{d}^{n+1}$

$$
\mathscr{J}_{\zeta}=\left\{I(\lambda)=\int_{\infty}^{\omega \infty}(\zeta t)^{v} \exp \left(-f_{\lambda}(\zeta t)\right) \frac{d t}{t} \mid v \in \mathscr{A}, \omega=e^{2 \pi i / d}\right\} .
$$

As in the introduction, for each $\zeta \in M_{d}^{n+1}$ we define the map

$$
\varphi_{\zeta}: \mathscr{V}_{\lambda} \longrightarrow \mathscr{F}_{\zeta}
$$

by

$$
\varphi_{\zeta}\left(t^{v}\right)=\int_{\infty}^{\omega \infty}(\zeta t)^{v} \exp \left(-f_{\lambda}(\zeta t)\right) \frac{d t}{t} .
$$

(Note that, although $\varphi_{\zeta}$ was initially defined on $\mathscr{L}$, it is actually defined on $\mathscr{V}_{\lambda}$ since $\Sigma D_{i} \mathscr{L} \subset \operatorname{ker} \varphi_{.}$) By definition, $\varphi_{\xi}$ is surjective for each $\zeta \in M_{d}^{n+1}$, so aside from the integral

$$
\varphi_{\zeta}(1)=\int_{\infty}^{\omega \infty} \exp \left(-f_{\lambda}(\zeta t)\right) \frac{d t}{t}
$$

each integral in $\mathscr{F}_{\zeta}$ may be regarded as the image under $\varphi_{\zeta}$ of a cohomology class on the variety $\boldsymbol{P}_{n-1}^{n}-X^{\phi}$ (or $X^{\phi}$ ). The strategy in proving the regular singularity of the integrals in $\mathscr{F}_{5}$ is as follows:

We show that $\partial / \partial \lambda$ acting on $\mathscr{F}_{\zeta}$ corresponds to $\sigma_{\lambda}$ acting on $\mathscr{V}_{\lambda}$ (Lemma 3). Since $\varphi_{\zeta}$ is surjective, the differential equation satisfied by each integral in $\mathscr{F}_{\zeta}$ is given by $\sigma_{\lambda}$ acting on its pre-image in $\mathscr{V}_{\lambda}$. Apart from the element $1, \sigma_{\lambda}$ acting on $\mathscr{C}_{\lambda}$ corresponds to $\partial / \partial \lambda$ acting on a quotient space of $H^{n-1}\left(X^{\phi}\right)$ (Lemma 2). By Griffiths' theorem, the periods on a variety have regular singularities, so it follows that, aside from $\varphi_{\zeta}(1)$, every integral in $\mathscr{F}_{\zeta}$ has regular singularities. The case $\varphi_{r}(1)$ is treated separately (Lemma 4 ).

LEMma 3. The following diagram commutes for each $\zeta \in M_{d}^{n+1}$ :

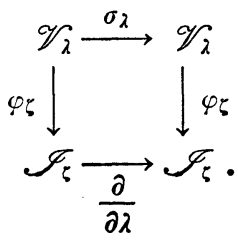

Proof. The proof is a standard computation which will be omitted. Note that a similar theorem is true for $\underset{2}{\mathscr{W}}$, a fact we will use later.

Let $I(\lambda) \in \mathscr{I}_{\zeta}, I(\lambda) \neq \varphi(1)$. Then: 


$$
\begin{aligned}
\frac{\partial I}{\partial \lambda} & =\frac{\partial}{\partial \lambda}\left(\varphi_{\zeta}\left(\xi_{v}\right)\right), \quad \xi_{v} \in \mathscr{W}_{\lambda} \\
& =\varphi_{\zeta}\left(\sigma_{\lambda}\left(\xi_{v}\right)\right) \\
& =\varphi_{\zeta}\left(\sum_{w \in \mathscr{S}-(0, \ldots, 0)} A_{v, w} \xi_{w}\right) \text { where } \sum_{w} A_{v, w} \xi_{w}
\end{aligned}
$$

is the system of differential equations satisfied $\xi_{v} \in \mathscr{W}_{\lambda}$. Hence, $\partial I / \partial \lambda=\sum_{w \in \cdots-(0, \cdots, 0)} A_{v, w} I_{w}$, so the system of differential equations satisfied by $I(\lambda) \in \mathscr{F}_{\xi}, I \neq \phi(1)$, is the same as that satisfied by a differential form on $\boldsymbol{P}_{n+1}^{n}-X^{\phi}$ (or $X^{\phi}$ ), so the integrals in $\mathscr{F}_{5}$ (aside from $\left.\varphi_{5}(1)\right)$ have regular singularities.

LEMMA 4. For each $\zeta \in M_{d}^{n+1}, \quad I(\lambda)=\int_{\infty}^{\omega \infty} \exp \left(-f_{\lambda}(\zeta t)\right) d t / t \quad h a s$ regular singularities.

Proof.

$$
\frac{\partial I}{\partial \lambda}=\int_{\infty}^{\omega \infty}\left(\frac{-\partial f}{\partial \lambda}\right) \exp \left(-f_{\lambda}(\zeta t)\right) \frac{d t}{t} \in \varphi\left(\mathscr{W}_{\lambda}\right)
$$

Hence, by the above argument, $\partial I / \partial \lambda$ has regular singularities and hence locally at most polynomial growth. Thus, locally its integral $I(\lambda)$ also has at most polynomial growth. It follows that $I(\lambda)$ has regular singularities.

The conclusion is that the integrals (*) in the $\mathscr{L}$ theory have regular singularities.

To draw the same conclusions about the integrals $\left({ }^{* *}\right)$ in the $\mathscr{L}^{s}$ theory, we need to compare their differential equations to those in the $\mathscr{L}$ theory. An outline of the method is as follows:

To pass to the $\mathscr{L}^{s}$ theory, we use Dwork's mapping $\Theta_{\lambda}$ which establishes an isomorphism between $\mathscr{K}_{\lambda}$ and $\mathscr{\mathscr { V }}_{\lambda}^{s}$, where $\mathscr{K}_{\lambda}$ is the dual space of $\mathscr{V}_{\lambda}$ (under a pairing to be defined). $\sigma_{\lambda}$ is a connection on $\mathscr{V}_{\lambda}^{s}$ and $\sigma_{\lambda}^{*}$, the dual operator, is a connection on $\mathscr{K}_{\lambda}$. Lemma 5 shows that the connection is consistent with $\Theta_{\lambda}$ and hence the connection matrix of $\sigma_{\lambda}$ acting on $\mathscr{C}_{\lambda}^{s}$ is the negative transpose of that of $\sigma_{\lambda}$ acting on $\mathscr{\mathscr { V }}_{\lambda}$. Since the system of differential equations satisfied by the integrals in the $\mathscr{L}^{8}$ theory is given by $\sigma_{\lambda}$ acting on $\mathscr{C O}_{\lambda}^{s}$ (same argument as in Lemma 3) and since the integrals in the $\mathscr{L}$ theory have regular singularities, it follows that the integrals $\left(^{* *}\right)$ in the $\mathscr{L}^{s}$ theory also have regular singularities.

To fill in the details of the above outline, we need to begin with some preliminaries on the dual theory. The dual theory is a $p$-adic theory, so for the definitions which follow to make sense, we must 
temporarily work over a $p$-adic field. Also, in order to define the dual pairing, we will interpret the monomial $X^{w} \in \mathscr{L}$ as $X_{0}^{w_{0}} X^{w}$ where $w_{0}=1 / d \sum_{i=1}^{n+1} w_{i}$.

Let $\Omega$ denote the completion of the algebraic closure of $\boldsymbol{Q}_{p}$, the field of $p$-adic numbers. Let $\mathscr{L}^{*}$ denote the $\Omega$-span of

$$
\left\{X^{-w} \mid \sum_{i=1}^{n+1} w_{i} \equiv 0(\bmod d) \text { and } w_{i} \geqq 0, i=1, \cdots, n+1\right\} \text {. }
$$

For $\xi \in \mathscr{L}$ (which we view as having coefficients in $\Omega$ ) and $\xi^{*} \in \mathscr{L}^{*}$, the product $\xi \cdot \xi^{*}$ is a well-defined element of $\mathscr{L} \cup \mathscr{L}^{*}$ provided certain growth conditions are satisfied ([2], p. 236). When this is the case, we may define the symbol

$$
\left\langle\xi^{*}, \xi\right\rangle=\text { the constant term of } \xi^{*}\left(X^{\prime}\right) \xi(X)
$$

where for $X=\left(X_{0}, X_{1}, \cdots, X_{n+1}\right), X^{\prime}=\left(-X_{0}, X_{1}, \cdots, X_{n+1}\right)$. Let $\mathscr{K}_{\lambda}$ denote the dual space of $\mathscr{C}_{\lambda}$ under this pairing.

For $w$ satisfying $\sum_{i=1}^{n+1} w_{i} \equiv 0(d)$, we define

$$
\gamma_{-}\left(X^{w}\right)= \begin{cases}X^{w} & \text { if each } w_{i} \leqq 0 \\ 0 & \text { otherwise }\end{cases}
$$

Dwork's $\Theta_{2}$ map, which allows us to pass from $\mathscr{K}_{\lambda}$ to $\mathscr{C}_{2}^{s}$, is defined as follows:

$$
\Theta_{\lambda}=\exp \left(f_{\lambda}(X)\right) \circ \sum_{i=1}^{n+1} X_{i} \frac{\partial}{\partial X_{i}} \circ \gamma_{-} \circ \exp \left(-f_{\lambda}(X)\right) .
$$

To compute the system of differential equations satisfied by the integrals in the $\mathscr{L}^{s}$ theory, we need to know how $\sigma_{2}$ behaves under the $\Theta_{\lambda}$ mapping.

LEMMA 5. The following diagram commutes:

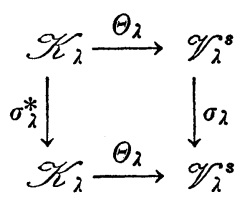

where $\sigma_{2}^{*}$ is the operator dual to $\sigma_{2}$ under the pairing defined above.

Proof. Under the pairing $\langle$,$\rangle defined earlier,$

$$
\sigma^{*}=\gamma_{-} \circ \sigma=\gamma_{-} \circ \exp \left(f_{\lambda}(X)\right) \circ \frac{\partial}{\partial \lambda} \circ \exp \left(-f_{\lambda}(X)\right) \text {. }
$$

Commutativity follows from a standard computation. 
To complete the proof of Theorem 2, we need to show that the connection matrix of $\sigma_{\lambda}$ acting on $\mathscr{V}_{\lambda}^{s}$ has regular singularities.

Suppose $\sigma_{\lambda}\left(\xi_{v}\right)=\sum_{v} A_{v, w} \xi_{w}$ where $\xi_{v}, \xi_{w} \in \mathscr{\mathscr { V } _ { 2 }}$. Then, since

$$
\left\langle\sigma^{*}\left(\xi_{u}^{*}\right), \xi_{v}\right\rangle=-\left\langle\xi_{u}^{*}, \sigma \xi_{v}\right\rangle=-A_{v, u},
$$

it follows that

$$
\sigma^{*}\left(\xi_{u}^{*}\right)=\sum_{v} B_{u, v} \xi_{v}^{*} \quad \text { where } \quad B_{u, v}=-A_{v, u} .
$$

Let $\eta \in \mathscr{V}_{\lambda^{*}}^{s}$. Then $\eta=\Theta\left(\xi_{u}^{*}\right)$ for some $\xi_{u}^{*} \in \mathscr{K}_{\lambda}$. By Lemma 5,

$$
\sigma(\eta)=\sum B_{u, v} \eta_{v}, \quad \eta_{v} \in \mathscr{Y}_{i}^{s} .
$$

The conclusion is that $\eta$ satisfies a system of differential equations with regular singularities, and since the integrals $\left({ }^{* *}\right)$ in the $\mathscr{L}^{s}$ theory satisfy the same system, they, too, have regular singularities. This concludes the proof of Theorem 2.

Note. We used Dwork's p-adic dual theory in the second part of the theorem in order to prove a classical result (i.e., a result over C). This could be avoided by using a result in [7], which expresses $\mathscr{C}_{\lambda}^{s}$ in terms of the $D_{i}$ 's acting on the middle dimensional cohomology of $X_{2}$ and its intersections with the axes $X_{1}=0, \cdots, X_{n+1}=0$. However, since the proof of this result is long, we have chosen to use Dwork's $p$-adic theory.

3. In this section we study the logarithmic growth of the integrals $\left(^{*}\right)$ and $\left({ }^{* *}\right)$. Since these integrals satisfy differential equations with regular singularities it follows that locally they are one of the following forms:

(1) $\varphi(\lambda)$, where $\varphi$ is a uniform function of $\lambda$,

(2) $\lambda^{\alpha} \varphi(\lambda)$ where $\alpha \in \boldsymbol{Q}[6]$,

(3) $\log ^{r}(\lambda) \varphi(\lambda)$ where $r \in \boldsymbol{Z}$, or

(4) sums of $\lambda^{\alpha} \log ^{r}(\lambda) \varphi(\lambda)$.

We are interested in finding an upper bound on $r$ for these integrals.

THEOREM 3. Locally the integrals

$$
\int_{\infty}^{\infty \infty}(\zeta t)^{v} \exp \left(-f_{\lambda}(\zeta t)\right) \frac{d t}{t}, \quad \zeta \in M_{d}^{n+1}, v \in \mathscr{A}
$$

and

$$
\int_{0}^{\infty}(\zeta t)^{v} \exp \left(-f_{\lambda}(\zeta t)\right) \frac{d t}{t}, \quad \zeta \in M_{d}^{n+1}, v \in \mathscr{A}^{\prime},
$$

have logarithmic singularities of order at most $n\left(n=1+\operatorname{dim} X_{\lambda}^{\phi}\right)$. 
Proof. By Griffiths' local monodromy theorem [6] it follows that locally the periods on the variety $X^{\phi}$ (of dimension $n-1$ ) have logarithmic singularities of order at most $n-1$. Hence, aside from the integral $\varphi_{\zeta}(1)=\int_{\infty}^{\omega \infty} \exp \left(-f_{\lambda}(\zeta t)\right) d t / t$, all of the integrals $\left(^{*}\right)$ in the $\mathscr{L}$ theory have local logarithmic singularities of order at most $n-1$. We settle the question of $\varphi_{\zeta}(1)$ as follows:

LEMMA 6. Locally, $I(\lambda)=\int_{\infty}^{\omega \infty} \exp \left(-f_{\lambda}(t)\right) d t / t$ has logarithmic growth of order at most $n$.

Proof. As in Lemma 4, $\partial I / \partial \lambda \in \varphi\left(\mathscr{W}_{\lambda}\right)$, so $\partial I / \partial \lambda$ has logarithmic singularities of order at most $n-1$. Since integrating $\log ^{n-1}(\lambda)$ gives at most $\log ^{n}(\lambda)$, the lemma follows.

To pass to the $\mathscr{L}^{s}$ theory, recall from Theorem 2 that the matrix giving the system of differential equations for the integrals in the $\mathscr{L}^{8}$ theory is the negative transpose of that in the $\mathscr{L}$ theory. Theorem 3 for the $\mathscr{L}^{\text {s }}$ theory is a consequence of the following general lemma:

Lemma 7. Suppose $Y^{\prime}=Y C$ where $Y, Y^{\prime}$ and $C$ are square matrices. Then if $Z$ satisfies $Z^{\prime}=-Z C^{t}$, it follows that locally $Z$ has the same order of logarithmic singularity as $Y$.

Proof. The proof is a standard computation in differential equations. The lemma and hence the theorem follow.

4. The original proof of the regularity of the integrals in the $\mathscr{L}$ theory uses Dwork's deformation theory to obtain explicit formulas for the integrals (*) as sums of periods on the variety $X_{\lambda}^{\phi}$. Since the formulas are interesting and in a special case show the relation of periods to hypergeometric functions, we outline briefly their derivation.

For $\lambda \in \Omega^{\nu}$, we define the deformation mapping

$$
T_{0, \lambda}: \mathscr{K}_{0} \longrightarrow \mathscr{K}_{\lambda}
$$

by

$$
T_{0, \lambda}=\gamma_{-} \circ \exp \left(f_{0}(X)-f_{\lambda}(X)\right) \text {. }
$$

This makes sense provided $\lambda$ is sufficiently close to 0 (in the $p$-adic sense) ([2], pp. 256-60). Let $\left\{\xi_{u, \lambda}\right\}_{u \in \mathscr{A}}$ be the basis of $\mathscr{K}_{\lambda}$ dual to $\left\{X^{u}\right\}_{u \in \mathscr{N}}$. Then relative to the bases $\left\{\xi_{u, 0}\right\}_{u \in \mathscr{N}}$ and $\left\{\xi_{u, \lambda}\right\}_{u \in \mathscr{N}}$, Katz shows that the matrix of $T_{0,2}$ satisfies the Picard-Fuchs differential equations for the variety $X_{\lambda}^{\phi}$ and hence (with the exception of the 
entries arising from $1 \in \mathscr{C}$ ) may be regarded as a matrix of periods on the variety $X_{i}^{\phi}$.

More explicitly, let $T \xi_{u, 0}^{*}=\sum_{v \epsilon \ldots} c_{u v} \xi_{v, \lambda}^{*}$ relative to the bases prescribed above. Then the $c_{u v}$ 's $(u, v \in \mathscr{A}-(0, \cdots, 0))$ may be regarded as periods on $X_{i}^{\phi}$. In [7], after a lengthy computation, an expression for the $c_{u v}$ 's in terms of the integrals $\left(^{*}\right)$ is given. Before stating the result we need to define the following gammalike function: for $u \in \boldsymbol{Z}, u \geqq 0$, let

$$
\widetilde{\Gamma}\left(\frac{u}{d}\right)=\int_{\infty}^{\omega \infty} e^{-t^{d}} t^{u} \frac{d t}{t}
$$

where $\omega=e^{2 \pi i / d}$ and the region of integration is the limit as $\rho \rightarrow 0$ along the path

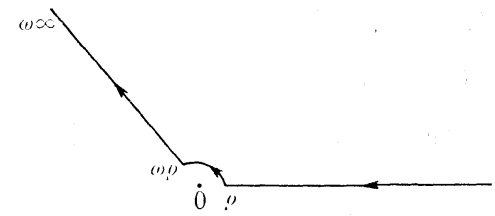

$\widetilde{\Gamma}$ can be shown to be well-defined. For $u \in \mathscr{A}$, let

$$
\widetilde{l}\left(\frac{u}{d}\right)=\prod_{i=1}^{n+1} \widetilde{\Gamma}\left(\frac{u_{i}}{d}\right)
$$

Then we have the following representation for periods $c_{u v}$ in terms of the integrals $\left(^{*}\right)[7]$ :

$$
c_{u v}=\tilde{l}\left(\frac{u}{d}\right)^{-1} \sum_{\zeta \in \mathbb{M} I_{d}^{n+1}} \zeta^{-u} \int_{\infty}^{\infty \infty}(\zeta t)^{v} \exp \left(-f_{\lambda}(\zeta t)\right) \frac{d t}{t}
$$

where $u, v \in \mathscr{A}$.

Inverting this expression leads to the desired formula for the integrals $\left(^{*}\right)$ as sums of periods $c_{u v}$. After noting that we only need to consider integrals of the form $\varphi_{\omega^{a}}\left(t^{v}\right)$ where $\omega=e^{2 \pi i / d}$ and $a \in A=$ $\left\{a=\left(a_{1}, \cdots, a_{n+1}\right) \mid \sum_{i=1}^{n+1} a_{i} \equiv 0(d)\right.$ and $\left.0 \leqq a_{i}<d, \quad i=1, \cdots, n+1\right\}$ $[7$, p. 42], it follows that

$$
\varphi_{\omega^{a}}\left(t^{v}\right)=\frac{\tilde{l}\left(\frac{a}{d}\right)}{d^{n+1}} \sum_{\substack{u_{1}, \ldots, u_{n} \\ 0 \leqq u_{j} \leqq d-1}} \omega^{\sum_{\imath=1}^{n} a_{\imath} u_{i}} c_{u, v}
$$

where $u, v \in \mathscr{A}$. Theorem 2 for the $\mathscr{L}$ theory follows from this formula.

5. We now compute an example to show the relation of periods 
on a variety to hypergeometric functions. As a result, we obtain the estimates on the growth of the periods independently of Griffith's work.

Let $f_{\lambda}(t)=t_{1}^{3}+t_{2}^{3}+t_{3}^{3}-3 \lambda t_{1} t_{2} t_{3}(\lambda \in C)$ so $f_{\lambda}(t)=0$ defines a family of curves in $\boldsymbol{P}^{2}(\boldsymbol{C})$ with singular fibres at $\lambda=1, e^{2 \pi i / 3}, e^{4 \pi i / 3}$ and $\infty$. From above,

$$
c_{u v}=\frac{1}{\tilde{l}\left(\frac{u}{3}\right)} \sum_{\zeta \in M_{3}^{3}} \zeta^{-u+v} H_{v}(\zeta)
$$

where $H_{v}(\zeta)=\int_{\infty}^{\omega \infty} t^{v-1} \exp \left(-f_{\lambda}(\zeta t)\right) d t$ for $\zeta \in M_{3}^{3}$. For $u, v \in \mathscr{A}-$ $\{0, \cdots, 0\}$, the $c_{u v}$ 's are periods on the variety $X_{2}^{\phi}$. Letting $\zeta=$ $\left(\omega^{a}, \omega^{b}, \omega^{c}\right)\left(\omega=e^{2 \pi i / d}\right)$, it follows that

$$
H_{v}(\zeta)=\left\{\begin{array}{lll}
H_{v}(1,1,1) & \text { if } & a+b+c \equiv 0(3) \\
H_{v}(\omega, 1,1) & \text { if } & a+b+c \equiv 1(3) \\
H_{v}\left(\omega^{2}, 1,1\right) & \text { if } & a+b+c \equiv 2(3)
\end{array}\right.
$$

Let $H_{v, \zeta}=H_{v}(\zeta, 1,1)$. Then a short computation shows that the $H_{v}$ 's may be written as hypergeometric functions as follows: For $a=0,1,2$,

$$
\begin{aligned}
H_{v, \omega^{a}}= & \widetilde{l}\left(\frac{v}{3}\right)_{3} F_{2}\left(\begin{array}{l}
\frac{v_{1}}{3}, \frac{v_{2}}{3}, \frac{v_{3}}{3} \\
\frac{1}{3}, \frac{2}{3}
\end{array} ; \lambda^{3}\right) \\
& +3 \lambda \omega^{a} \widetilde{l}\left(\frac{v+1}{3}\right)_{3} F_{2}\left(\begin{array}{l}
\frac{v_{1}+1}{3}, \frac{v_{2}+1}{3}, \frac{v_{3}+1}{3} ; \lambda^{3} \\
\frac{2}{3}, \frac{4}{3}
\end{array}\right) \\
& +(3 \lambda)^{2} \omega^{2 a} \tilde{l}\left(\frac{v+2}{3}\right)_{3} F_{2}\left(\frac{\frac{v_{1}+2}{3}, \frac{v_{2}+2}{3}, \frac{v_{3}+2}{3}}{\frac{5}{3}} ; \lambda^{3}\right)
\end{aligned}
$$

where the hypergeometric function

$$
{ }_{3} F_{2}\left(\begin{array}{c}
\alpha, \beta, \gamma \\
\delta, \varepsilon
\end{array} ; \lambda^{3}\right)=\sum_{m=0}^{\infty} \frac{(\alpha)_{m}(\beta)_{m}(\gamma)_{m}}{(\delta)_{m}(\varepsilon)_{m}(1)_{m}} \lambda^{3 m}
$$

Since

$$
c_{u v}= \begin{cases}9 \tilde{l}\left(\frac{u}{3}\right)^{-1}\left\{H_{v, 1}+\omega^{2\left(u_{1}-v_{1}\right)} H_{v, \omega}+\omega^{u_{1}-v_{1}} H_{v, \omega^{2}}\right\} & \text { if } u_{1}-v_{1} \equiv u_{2}-v_{2} \\ 0 & \text { otherwise }\end{cases}
$$


it follows that:

$$
\begin{aligned}
& \left(\frac{3^{3} \tilde{l}\left(\frac{v}{3}\right)}{\tilde{l}\left(\frac{u}{3}\right)^{3}} F_{2} \mid \begin{array}{l}
\frac{v_{1}}{3}, \frac{v_{2}}{3}, \frac{v_{3}}{3} \\
\frac{1}{3}, \frac{2}{3}
\end{array} ; \lambda^{3}\right) \text { if } u_{1}-v_{1} \equiv 0(3) \text { and } u_{2}-v_{2} \equiv 0(3) \\
& c_{u v}=\left\{\begin{array}{l}
\frac{3^{4} \lambda \tilde{l}\left(\frac{v+1}{3}\right)}{\tilde{l}\left(\frac{u}{3}\right)} F_{3}\left(\begin{array}{ll}
\frac{v_{1}+1}{3}, & \frac{v_{2}+1}{3}, \frac{v_{3}+1}{3} ; \lambda^{3}
\end{array}\right) \\
\quad \text { if } u_{1}-v_{1} \equiv 1(3) \text { and } u_{2}-v_{2} \equiv 1(3)
\end{array}\right. \\
& \frac{3^{5} \lambda^{2} \tilde{l}\left(\frac{v+2}{3}\right)}{\tilde{l}\left(\frac{u}{3}\right)} F_{2} F_{2}\left(\begin{array}{c}
\frac{v_{1}+2}{3}, \frac{v_{2}+2}{3}, \frac{v_{3}+2}{3} \\
\frac{4}{3}, \frac{5}{3}
\end{array} ; \lambda^{3}\right) \\
& \text { if } \quad u_{1}-v_{1} \equiv 2(3) \quad \text { and } \quad u_{2}-v_{2} \equiv 2(3) \\
& 0 \text { otherwise . }
\end{aligned}
$$

The fact that the $c_{u, v}$ 's are expressed in terms of hypergeometric functions of the type ${ }_{m+1} F_{m}$ (Pochhammer hypergeometric functions) shows that they have regular singularities. Since the hypergeometric functions involved are of the form ${ }_{3} F_{2}$, the indicial polynomial has at most three roots, so $c_{u v}$ can have logarithmic singularities of order at most two.

Note further that when $u, v \in \mathscr{A} \cap \mathscr{A}^{\prime}$, (i.e., $0<u, v<d$ ) there is cancellation in ${ }_{3} F_{2}$ so it becomes ${ }_{2} F_{1}$, and in this case there are logarithmic singularities of order at most one. This is to be expected, since it follows from Katz's thesis [4] that such $c_{u v}$ are periods on $X_{\lambda}$.

This computation can be generalized to the class of hypersurfaces defined by the vanishing of the form:

$$
f_{\lambda}(X)=X_{1}^{d}+\cdots+X_{n+1}^{d}+\lambda X_{1}^{w_{1}} \cdots X_{n+1}^{w_{n+1}}, \quad \lambda \in C .
$$

In this special case the statements about the regular singularity and logarithmic growth of the periods (Theorems 2 and 3) follow from classical hypergeometric theory.

6. We conclude this paper with an example showing the necessity of the hypothesis that $f_{\lambda}(t)$ be homogeneous. We do this by dehomogenizing the family of cubic curves in the previous example and showing that the resulting integrals have irregular singularities at infinity. 
Let $f_{\lambda}(t)=t_{1}^{3}+t_{2}^{3}+t_{3}^{3}-3 \lambda t_{1} t_{2} t_{3}$. Letting $y_{i}=t_{i} / t_{3}, i=1$, 2 , we obtain the inhomogeneous polynomial $\widetilde{f}_{\lambda}(y)=1+y_{1}^{3}+y_{2}^{3}-3 \lambda y_{1} y_{2}$. Proceeding as above, the integral

$$
I(\lambda)=\int_{\infty}^{\omega \infty} \int_{\infty}^{\omega \infty} y_{1}^{v_{1}} y_{2}^{v_{2}} \exp \left(-\widetilde{f}_{\lambda}(y)\right) \frac{d y_{1}}{y_{1}} \frac{d y_{2}}{y_{2}}
$$

reduces to a sum of hypergeometric functions of the type ${ }_{2} F_{2}$. Since each ${ }_{2} F_{2}$ satisfies a third-order differential equation and since there are only two exponents at $\infty$, each ${ }_{2} F_{2}$ has irregular singularities at infinity.

This example generalizes readily to inhomogeneous polynomials of the type

$$
g_{\lambda}(y)=y_{1}^{d}+\cdots+y_{n+1}^{d}+\lambda y_{1}^{r_{1}} \cdots y_{n+1}^{r_{n+1}}
$$

where $r_{i} \geqq 0, i=1, \cdots, n+1$. As above, the integral

$$
I(\lambda)=\int_{\infty}^{\omega \infty} y^{v} \exp \left(-g_{\lambda}(y)\right) \frac{d y}{y}
$$

may be expressed in terms of hypergeometric functions of the type ${ }_{\alpha} F_{d-1}$ where $\alpha=\sum_{i=1}^{n+1} r_{i}$. These have regular singularities exactly when $\sum_{i=1}^{n+1} r_{i}=d$, i.e., if and only if $g_{\lambda}(y)$ is homogeneous.

\section{REFERENCES}

1. B. Dwork, On the Zeta function of a hypersurface, Publ. Math. I.H.E.S., 12 (1962), $5-85$.

2. On the Zeta function of a hypersurface: II, Ann. of Math., 80 (1964), $227-299$.

3 - Bessel functions as p-adic functions of the argument, Duke Math. J., 41 (1974), 711-738.

4. N. Katz, On the differential equations satisfied by period matrices, Publ. Math. I.H.E.S., 35 (1968), 223-258.

5. - On the intersection matrix of a hypersurface, Ann. E.N.S., 4-série, tome 2, fasc., 4 (1969), 583-598.

6. - Nilpotent connections and the monodromy theorem: applications of a result of Turritin, Publ. Math. I.H.E.S., 39 (1970), 175-232.

7. E. Stevenson, Integral Representations of Algebraic Cohomology Classes on Hypersurfaces, Princeton thesis, 1975.

Received October 5, 1976.

UNIVERSITY OF COLORADO

BOULDER, CO 80302 


\section{PACIFIC JOURNAL OF MATHEMATICS}

\section{EDITORS}

RICHARD ARENS (Managing Editor)

University of California

Los Angeles, California 90024

C. W. CURTIS

University of Oregon

Eugene, OR 97403

C. C. MOORE

University of California

Berkeley, CA 94720

\section{J. DugundJI}

Department of Mathematics University of Southern Californıa Los Angeles, California 90007

R. FinN AND J. Milgram Stanford University Stanford, California 94305

\section{ASSOCIATE EDITORS}

E. F. BECKENBACH

B. H. NEUMANN

F. WOLF

K. YOSHIDA

\section{SUPPORTING INSTITUTIONS}

UNIVERSITY OF BRITISH COLUMBIA CALIFORNIA INSTITUTE OF TECHNOLOGY UNIVERSITY OF CALIFORNIA MONTANA STATE UNIVERSITY UNIVERSITY OF NEVADA, RENO NEW MEXICO STATE UNIVERSITY OREGON STATE UNIVERSITY UNIVERSITY OF OREGON OSAKA UNIVERSITY
UNIVERSITY OF SOUTHERN CALIFORNIA STANFORD UNIVERSITY UNIVERSITY OF TOKYO UNIVERSITY OF UTAH WASHINGTON STATE UNIVERSITY UNIVERSITY OF WASHINGTON AMERICAN MATHEMATICAL SOCIETY NAVAL WEAPONS CENTER 


\section{Pacific Journal of Mathematics}

\section{Vol. 71, No. $1 \quad$ November, 1977}

Charalambos D. Aliprantis and Owen Sidney Burkinshaw, On universally complete Riesz spaces ............................. 1

Stephen Richard Bernfeld and Jagdish Chandra, Minimal and maximal solutions of nonlinear boundary value problems .................

John H. E. Cohn, The length of the period of the simple continued fraction of

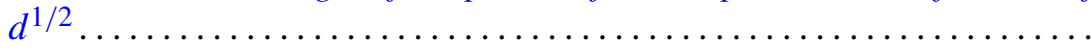

Earl Vern Dudley, Sidon sets associated with a closed subset of a compact abelian group .................................... 33

Larry Finkelstein, Finite groups with a standard component of type $J_{4} \ldots \ldots$

Louise Hay, Alfred Berry Manaster and Joseph Goeffrey Rosenstein, Concerning partial recursive similarity transformations of linearly ordered sets .......................................

Richard Michael Kane, On loop spaces without $p$ torsion. II ............

William A. Kirk and Rainald Schoneberg, Some results on

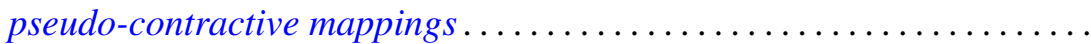

Philip A. Leonard and Kenneth S. Williams, The quadratic and quartic character of certain quadratic units. I. .

Lawrence Carlton Moore, A comparison of the relative uniform topology and the norm topology in a normed Riesz space .................

Mario Petrich, Maximal submonoids of the translational hull 119

Mark Bernard Ramras, Constructing new R-sequences . . .

Dave Riffelmacher, Multiplication alteration and related rigidity properties

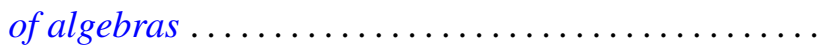

Jan Rosiński and Wojbor Woyczynski, Weakly orthogonally additive functionals, white noise integrals and linear Gaussian stochastic processes.

Ryōtarō Satō, Invariant measures for ergodic semigroups of operators

Peter John Slater and William Yslas Vélez, Permutations of the positive integers with restrictions on the sequence of differences...

Edith Twining Stevenson, Integral representations of algebraic cohomology classes on hypersurfaces ........................

Laif Swanson, Generators of factors of Bernoulli shifts . 